\title{
On the Common Mode Response of Fully Differential Circuits
}

\author{
M. Gasulla, O. Casas and R. Pallàs-Areny \\ Divisió d'Instrumentació i Bioenginyeria, Dept. d'Enginyeria Electrònica \\ Universitat Politècnica de Catalunya, c/Jordi Girona 1-3, Edifici C-4, 08034 Barcelona, SPAIN \\ Phone 34-3-401-6766 Fax 34-3-401-6756 e-mail: elerpa@eel.upc.es \\ WWW: http://petrus.upc.es/wwwdib/homepageuk.html
}

\begin{abstract}
Differential circuits are often described by their differential gain and common mode rejection ratio (CMRR). This approach, however, neglects the effect that the common mode signal has on the transient response and stability of the circuit. This work shows that the actual behavior of differential circuits in front of common mode voltages is completely described by the common-to-differential mode gain and the commonmode gain. The CMRR is useful to assess common mode errors in the frequency domain, but some circuits that achieve a large CMRR have long transients or are unstable.
\end{abstract}

\section{Introduction}

Differential circuits are suited to process signals from sensor bridges and differential sensors. Often, a differential amplifier yields a single-ended voltage that undergoes further processing (demodulation, filtering) before sampling and analog-to-digital conversion. However, it has been shown that the later the differential to single-ended conversion in the measurement chain, the higher the common mode rejection ratio (CMRR) [1][2]. Moreover, because differential signals have increased immunity to interference, it is convenient not to convert them to singleended signals. The increasing availability of differential analog-to-digital converters (ADC) enables this approach.

Fully differential circuits (differential input and output) are described by four transfer functions (Figure 1). The differential and common mode outputs are [3]

$$
\begin{aligned}
& V_{\mathrm{oD}}=G_{\mathrm{DD}} V_{\mathrm{iD}}+G_{\mathrm{DC}} V_{\mathrm{iC}} \\
& V_{\mathrm{oC}}=G_{\mathrm{CD}} V_{\mathrm{iD}}+G_{\mathrm{CC}} V_{\mathrm{iC}}
\end{aligned}
$$

where $V_{\mathrm{iD}}$ and $V_{\mathrm{iC}}$ are, respectively, the (transforms of the) differential and common mode input voltages. Because the information is embedded in the differential voltage, we ideally wish $G_{\mathrm{DC}}=0$. Having $G_{\mathrm{CD}}=0$ is also convenient, but it is less important if the following stage rejects common mode signals. In practice, however, $G_{\mathrm{DC}} \neq 0$ and we define the CMRR as

$$
\mathrm{CMRR}=\frac{G_{\mathrm{DD}}}{G_{\mathrm{DC}}}
$$

The differential output voltage is then [3][4]

$$
V_{\mathrm{oD}}=G_{\mathrm{DD}}\left(V_{\mathrm{iD}}+\frac{V_{\mathrm{iC}}}{\mathrm{CMRR}}\right)
$$

which describes the effect of the common mode voltage as an additive error, provided the common and differential mode voltages are independent from each other. To further simplify matters, although the CMRR is frequency dependent, usually the error described by (3) is calculated by using the CMRR value in the frequency band of interest, namely that of $V_{\mathrm{iD}}$. Equation (3) also applies to circuits with differential input and single-ended output, in which case $G_{\mathrm{DD}}=G_{\mathrm{D}}, G_{\mathrm{DC}}=G_{\mathrm{C}}$.

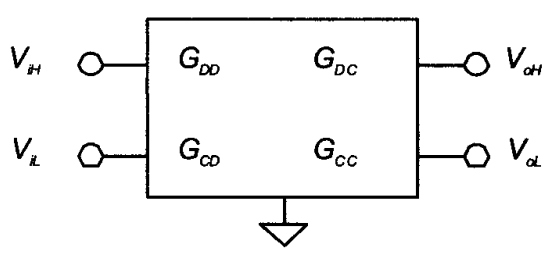

Figure 1. Fully differential circuits are described by four transfer functions.

Although eqn. (3) is extensively used, it does not describe the actual circuit behavior to the common mode input voltage $v_{\mathrm{ic}}$. A large CMRR, for example, suggests a 
small error due to $v_{\mathrm{iC}}$, but if $G_{\mathrm{DC}}$ is unstable the circuit will not work [5][6]. Also, if $G_{\mathrm{DC}}$ has a long transient response, it cannot be explained from $G_{\mathrm{DD}}$ and eq. (3).

This work shows that the CMRR does not fully describe the common mode response of differential circuits and that $G_{\mathrm{DC}}$ and $G_{\mathrm{CC}}$ must be designed to achieve the desired behavior to common mode signals.

\section{Theoretical analysis}

\subsection{Long transient response due to $\mathrm{G}_{\mathrm{DC}}$}

We have investigated the actual influence of $G_{D C}$ in several fully differential circuits. Figure 2, for example, shows a fully differential high-pass filter. $R_{\mathrm{c}}=0 \Omega$ yields an uncoupled filter. Adding $R_{\mathrm{c}} \gg R_{1}$ improves the lowfrequency CMRR by $2 R_{\mathrm{c}} / R_{1}$ [7]. An infinite $R_{\mathrm{c}}$ would yield infinite CMRR, but $R_{\mathrm{c}}$ must be finite to bias the following amplifier. Considering component tolerances in a worst case situation,

$$
\begin{array}{ll}
R_{1}=R(1+\alpha) & C_{1}=C(1+\beta) \\
R_{1}^{\prime}=R(1-\alpha) & C_{1}^{\prime}=C(1-\beta)
\end{array}
$$

yields

$$
G_{D C} \approx \frac{2(\alpha+\beta) R C s}{(1+R C s)\left(1+\left(R+2 R_{c}\right)\right) C s}
$$

where we have assumed $R_{\mathrm{c}} \gg R$. From (5), the differential mode output when applying a common mode step voltage of amplitude $A$ is

$$
V_{0} \approx A(\alpha+\beta) \frac{R}{R_{c}}\left[e^{-t /\left(R+2 R_{c}\right) C}-e^{-t / R C}\right]
$$

If $R_{\mathrm{c}}=0$, circuit analysis yields

$$
V_{0}=A\left(e^{-t / R_{1} C_{1}}-e^{-t / R_{1}^{\prime} C_{1}^{\prime}}\right)
$$

Figure 3 is a normalized plot of (6) and (7) showing that the output voltage does not correspond to the step response of a high-pass filter $\left(G_{\mathrm{DD}}\right)$ but to the bandpass filter described by (5). The theoretical peak amplitude and the time needed to reach it are

$$
\begin{aligned}
& A_{p} \approx 2 A(\alpha+\beta) e^{-1} \\
& t_{p} \approx R C
\end{aligned}
$$

for the uncoupled filter and

$$
\begin{aligned}
& A_{p} \approx A(\alpha+\beta) \frac{R}{R_{c}} \\
& t_{p} \approx R C \ln \left(\frac{2 R_{c}}{R}\right)
\end{aligned}
$$

for the coupled filter when $R_{\mathrm{c}} \gg R$. Increasing $R_{\mathrm{c}}$ reduces $A_{\mathrm{p}}$ and increases CMRR, both of which are desirable, but also lengthens $t_{\mathrm{p}}$ and the decaying exponential tail, which are undesirable. For example, $R_{1}=100 \mathrm{k} \Omega, C_{1}=1 \mu \mathrm{F}\left(f_{\mathrm{c}}=\right.$ $1.59 \mathrm{~Hz}), A=1 \mathrm{~V}, \alpha=5 \%$ and $\beta=10 \%$ yield $A_{\mathrm{p}}=110 \mathrm{mV}$ and $t_{\mathrm{p}}=100 \mathrm{~ms}$ for the uncoupled filter. However, at $t=1 \mathrm{~s}$ the output voltage is only $163 \mu \mathrm{V}$. If $R_{\mathrm{c}}=1 \mathrm{M} \Omega$ the CMRR increases by $26 \mathrm{~dB}$ and $A_{\mathrm{p}}$ decreases to about $15 \mathrm{mV}$ but $\mathrm{t}_{\mathrm{p}}$ increases to $300 \mathrm{~ms}$. Worse yet, since the exponential tail is determined by $2 R_{\mathrm{c}} C$, the output voltage after $1 \mathrm{~s}$ is $9.3 \mathrm{mV}$, hence 57 times larger than that of the uncoupled filter. The output decreases to $163 \mu \mathrm{V}$ at $t=9.5 \mathrm{~s}$ when $R_{\mathrm{c}}=1 \mathrm{M} \Omega$, and at $t=45 \mathrm{~s}$ when $R_{\mathrm{c}}=10 \mathrm{M} \Omega$. Therefore, the CMRR has improved at the cost of a slower transient response.

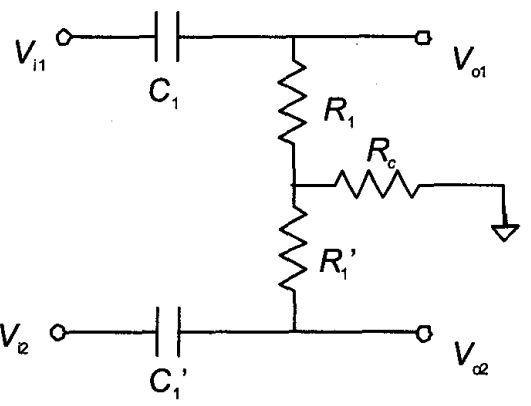

Figure 2. Fully differential high-pass filter. $R=0$ $\Omega$ yields an uncoupled filter.

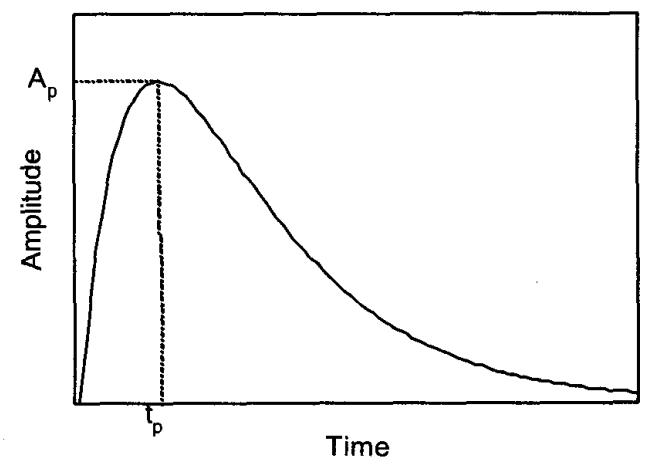

Figure 3. Common mode step response of the fully differential filter in Figure 2. 
Figure 4 shows a differential filter that does not need any grounded resistor to provide a bias path for the following amplifier. The input common mode voltage yields an output common mode voltage with the same amplitude $\left(G_{\mathrm{Cc}}=1\right)$ and cannot yield any differential output voltage. Hence, $G_{\mathrm{DC}}=0$ and $\mathrm{CMRR}=\infty$. The frequency response for the differential voltage can be designed to be the same as in Figure 2. Therefore, circuits with the same differential response can have quite different common mode response.

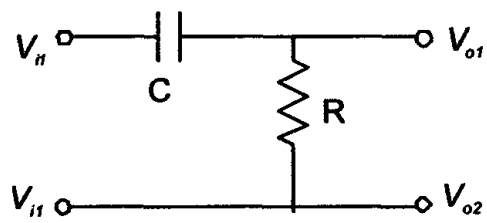

\section{Figure 4. Asymmetrical high-pass filter.}

\subsection{Circuit instability due to $G_{\mathrm{CC}}$}

Some circuits with high CMRR are unstable. Da Cunha [5] built a fully differential, second-order, low-pass filter based on a common Sallen and Key circuit that revealed to be oscillation-prone. We have analyzed that circuit and traced its instability to $G_{\mathrm{OC}}$. The CMRR would not explain that oscillation. Splitting capacitors and adding a small capacitance to ground $\left(C_{c}\right.$ in Figure 5) stabilizes the circuit at the cost of a reduced CMRR. If capacitors other than $C_{c}$ have nominal value $C$ and tolerance $\beta$, and resistors have nominal value $R$ and tolerance $\alpha$, when $C_{\mathrm{c}} \ll C$ the CMRR is

$$
\mathrm{CMRR} \approx-\frac{1}{2 R C_{c}(\alpha+\beta)} \frac{1+R C_{c} s+R^{2} C C_{c} s^{2}}{(1+R C s) s}
$$

and $\mathrm{G}_{\mathrm{cc}}$ is

$$
G_{C C} \approx \frac{1}{1+R C_{c} s+R^{2} C \frac{C_{c}}{2} s^{2}}
$$

which has resonance with amplitude $M_{r} \approx \sqrt{ } C /\left(2 C_{c}\right)$ at $\omega \approx$ $1 / \mathrm{R} \sqrt{ }\left(C C_{c} / 2\right)$. If $C_{\mathrm{c}}$ is close to zero, $M_{\mathrm{r}}$ tends to infinite, thus rendering the circuit unstable. Increasing $C_{c}$ reduces $M_{\mathrm{r}}$ (and $\omega$ ) but also the CMRR at low frequencies.

White [6] analyzed oscillation in the three-op-amp instrumentation amplifier, traced it to the common mode gain (here termed $G_{\mathrm{CC}}$ ) and solved it by a technique similar to that in Figure 5.

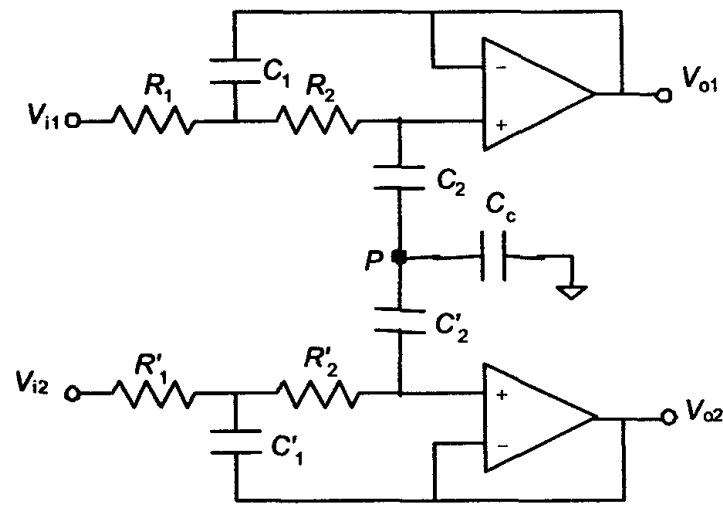

Figure 5. Fully differential, second-order, lowpass filter.

\section{Experimental results and discussion}

We have built the circuit in Figure 2 with $R_{1}=R_{\mathrm{I}}{ }^{\prime}=100$ $\mathrm{k} \Omega$ and $C_{1}=C_{1}{ }^{\prime}=1 \mu \mathrm{F}$. $R_{\mathrm{c}}$ was $0 \Omega$ (uncoupled filter), 1 $\mathrm{M} \Omega$, and $10 \mathrm{M} \Omega$. The circuit output was connected to an instrumentation amplifier (INA114) that provided gain (100), low output impedance, and a single-ended output. Figure 6 shows the amplifier output voltage when applying a $1 \mathrm{~V}$ common-mode step voltage and $R_{\mathrm{c}}=0 \Omega$ and $1 \mathrm{M} \Omega$. The uncoupled filter yields $A_{\mathrm{p}}=3.56 \mathrm{~V}$ and $t_{\mathrm{p}}=100 \mathrm{~ms}$, which, according to (8), implies $\alpha+\beta=4.8 \%$. When $R_{\mathrm{c}}=1 \mathrm{M} \Omega, A_{\mathrm{p}}$ decreases to $0.35 \mathrm{~V}, t_{\mathrm{p}}$ increases to $320 \mathrm{~ms}$, and the exponential decay is far slower. From $t=500 \mathrm{~ms}$ on, the output voltage is larger than that of the uncoupled filter. At $t=1 \mathrm{~s}$, the output is still $0.27 \mathrm{~V}$. The filter in Figure 4 yields a zero output, as expected.

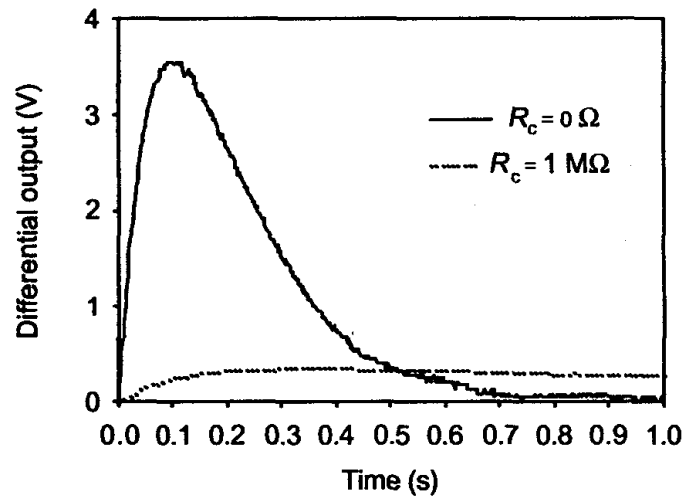

Figure 6. Output voltage for a passive differential filter (Fig. 2) followed by an INA114 instrumentation amplifier $(G=100)$, when 


\section{applying a $1 \mathrm{~V}$ common mode input voltage.}

Figure 7 shows the CMRR for the filters in Figures 2 and 4 when connected to the INA114 $(G=100)$. The CMRR for the circuit in Figure 2 improves when $R_{\mathrm{c}}$ is large. The CMRR measured at high frequencies is that of the INA114, because it is smaller than that of the preceding filter. The filter in Figure 4 yields the best CMRR, as predicted.

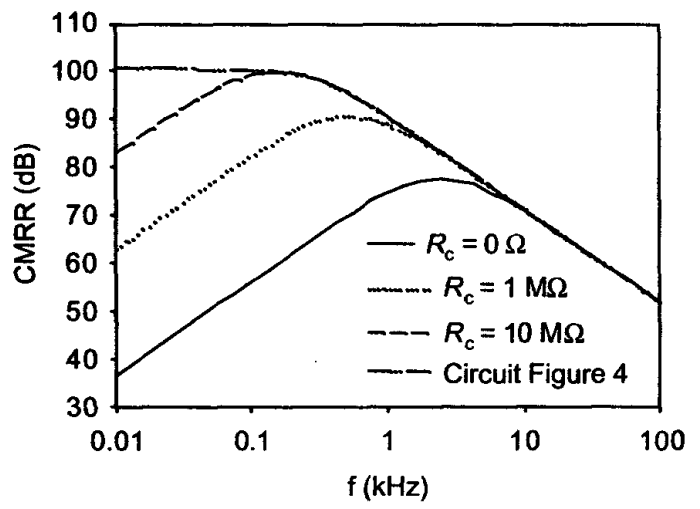

Figure 7. CMRR of the filters in Figures 2 and 4.

We have implemented the filter in Figure 5 by using TL072 op amps and designing $R_{1}=R_{1}{ }^{\prime}=R_{2}=R_{2}{ }^{\prime}=1.6 \mathrm{k} \Omega$, $C_{1}=C_{1}{ }^{\prime}=C_{2}=C_{2}{ }^{\prime}=1 \mu \mathrm{F}$, thus resulting in a $-3 \mathrm{~dB}$ corner frequency of $1 \mathrm{kHz}$. Grounding $P$ yields an uncoupled filter. Figure 8 shows the experimental $G_{\propto C}$ obtained for the uncoupled filter and for the coupled filter with $C_{\mathrm{c}}=100 \mathrm{nF}$, $10 \mathrm{nF}$, and $100 \mathrm{pF}$. The resonance peak and frequency decrease for large $C_{C}$. However, a large $C_{C}$ reduces the CMRR (Figure 9). Hence, there is a trade off between stability and CMRR, as anticipated by the theoretical analysis.

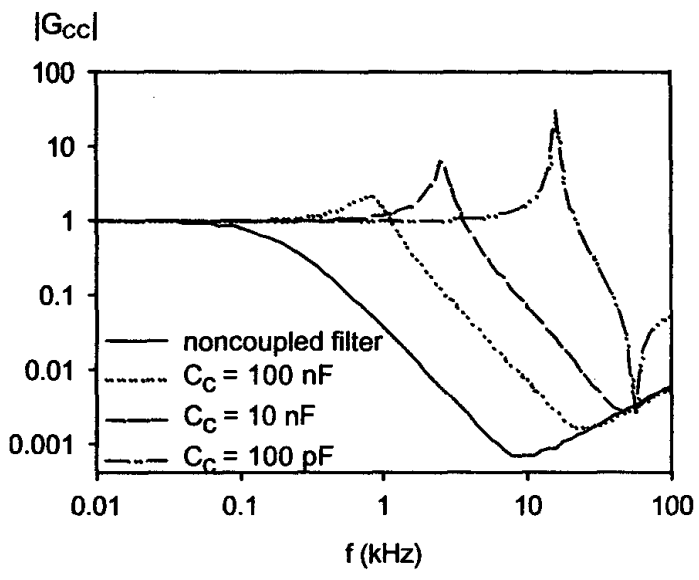

Figure 8. $G_{c c}$ for the filter in Figure 5.

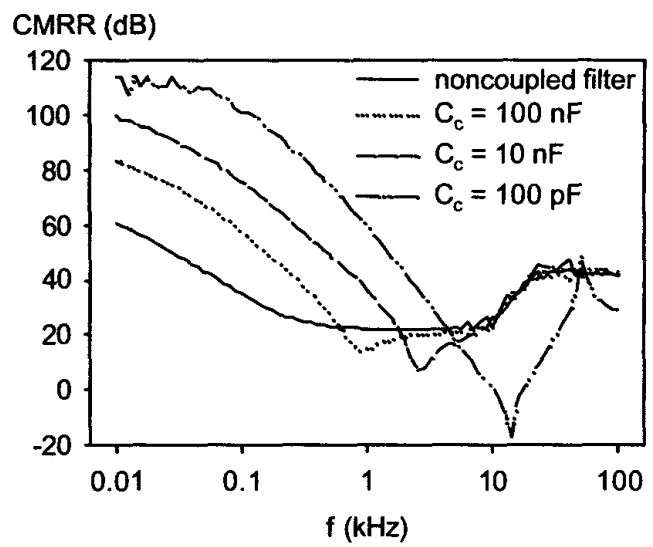

Figure 9. CMRR for the filter in Figure 5.

\section{Conclusion}

The common mode response of differential circuits is not fully described by the CMRR but by the common-todifferential mode gain $\left(G_{\mathrm{DC}}\right)$ and the common mode gain $\left(G_{\mathrm{OC}}\right)$. Their analysis reveals shortcomings such as long transient response and potential instability. Equation (3) is still useful to assess common mode errors in the frequency domain, but some circuits that achieve a large CMRR have long transients or are unstable.

\section{Acknowledgement}

This work has been funded by the Spanish CICYT, Project TAP99-0742.

\section{$\underline{\text { References }}$}

[1] R. Pallás-Areny and O. Casas, "A novel differential synchronous demodulator for AC signals," IEEE Trans. Instrum. Meas., vol. 45, pp. 413-416, Apr. 1996.

[2] M. Gasulla-Forner, J. Jordana-Barnils, R. Pallás-Areny, and J. M. Torrents, " The floating capacitor as a differential building block", IEEE Trans. Instrum. Meas., vol. 47, pp. 26-29, Feb. 1998.

[3] R. Pallás-Areny and John G. Webster, Analog Signal Processing. New York: John Wiley \& Sons, 1999.

[4] W. Kester (ed.). Practical Design Techniques for Sensor Signal Conditioning. Norwood, MA: Analog Devices, 1999.

[5] John M. da Cunha, "A compact and flexible signal conditioning system for data acquisition," Hewlett-Packard Journal, pp. 9-15, Oct. 1994.

[6] D. Rod White, "Phase compensation of the three op amp 
instrumentation amplifier," IEEE Trans. Instrum. Meas., vol. 36, pp. 842-844, Sept. 1987.

[7] O. Casas and R. Pallás-Areny, "Basics of analog differential filters," IEEE Trans. Instrum. Meas., vol. 45, pp. 275-279, Feb. 1996. 